\title{
Immunohistochemical profile of long-standing traumatic retinal detachment in atrophic globe in a young patient
}

\author{
SHILIANG LIU, YUANYUAN CHEN, ZHEN CHEN, YIQIAO XING and YIN SHEN \\ Eye Center, Renmin Hospital of Wuhan University, Wuhan, Hubei 430060, P.R. China
}

Received April 11, 2016; Accepted March 10, 2017

DOI: $10.3892 /$ etm.2018.6497

\begin{abstract}
Photoreceptor cell death is the ultimate cause of irreversible vision loss in retinal detachment (RD). The present study aimed to investigate the retinal changes in a case of long-standing traumatic RD in a young patient. The RD-induced atrophic globe was examined following enucleation. A control eye acquired from a deceased donor (normal histology; age- and sex-matched) was evaluated correspondingly. Frozen sections of retina tissue were assessed by immunofluorescence staining. The atrophic retina demonstrated structural disruption along with reduction in the retinal outer nuclear layer/inner nuclear layer thickness ratio. Photoreceptor degeneration was noted with complete loss of the outer segment of short-wavelength sensitive (S) cones. In addition, Müller cell hypertrophy was observed across the retinal nuclear layers. These results indicate that RD without successful medical treatment may lead to retinal atrophy associated with disruption of retinal integrity, dramatic $\mathrm{S}$ cones loss and subretinal gliosis. Further clarifications of the mechanisms underlying photoreceptor cell death and glial cell reprogramming may facilitate the design of novel therapeutic strategies for RD.
\end{abstract}

\section{Introduction}

Retinal detachment (RD) is an important cause of blindness and visual impairment worldwide (1). There are four major types of RD, including rhegmatogenous, exudative or serous, tractional and combined tractional-rhegmatogenous RD (2). Among all risk factors, including severe myopia, retinal tears, trauma, proliferative retinopathy and cataract surgery complications, trauma accounts for $11 \%$ of cases in adults affected by RD (3). RD refers to a severe disorder in which the retina separates from the underlying support tissue (4). Extensive death of photoreceptor cells occurs once they are physically

Correspondence to: Dr Yin Shen, Eye Center, Renmin Hospital of Wuhan University, 238 Jiefang Road, Wuhan, Hubei 430060, P.R. China

E-mail: yinshen@whu.edu.cn

Key words: retinal detachment, $\mathrm{S}$ cones, Müller cells hypertrophy separated from the retinal pigment epithelium (RPE) and choroidal vessels, a source of oxygen and nourishment for photoreceptor cells (5). In human RD caused by trauma, early events of photoreceptor apoptosis have been detected at $8 \mathrm{~h}$, peaking at $48 \mathrm{~h}$ (6) and dropping to a low level after 7 days (7). However, the irreversible loss of photoreceptor cells may continue as long as RD persists. As a result, although retinal surgery can successfully reattach the retina (8), visual acuity is not always restored.

Numerous histological and clinical studies have highlighted the biological process involved in RD-induced photoreceptor change. It has been demonstrated that short-wavelength sensitive (S) cones are more susceptible to damage compared with middle (M)-to long (L)-wavelength sensitive cones (9). Furthermore, RD seems to produce more damage to the cones than to the rods (10). In long-term RD, the majority of surviving cones failed to be labeled by almost all reliable markers, while rod photoreceptors continued to express most rod markers as long as they were alive (11). This may explain a faster rod vision rebuilding and specific color vision defects subsequent to successful reattachment surgeries. Furthermore, subretinal gliosis caused by Müller cells is involved in irreversible photoreceptor loss following RD (12). The functional outcome of proliferative vitreoretinopathy (PVR) is determined by the balance between protective and destructive repair mechanisms (13).

Although the pathophysiology of RD is better understood at present, the disease management remains an issue. Traumatic RD in pediatric patients is often found to be more challenging compared with that in an adult (14), and the anatomical success of surgery in children is much lower than in adults (15). Additional complications in long-standing $\mathrm{RD}$, including PVR, cataract and deprivation amblyopia, may further aggravate the symptoms and lead to atrophy $(16,17)$. Overactivity of Müller cells may be the cause of poor outcome in young patients undergoing RD medical treatment. Therefore, the prevention of PVR is indispensable for the success of RD repair (13). A systematic search for preoperative PVR risk factors is essential to provide valuable insight into a suitable, personalized therapeutic strategy.

Immunohistochemistry is important in establishing potential mechanisms and precise cellular responses following RD. To date, only a limited number of studies on human tissue of detached retina have been reported $(7,9)$. Therefore, the aim of the present study was to evaluate the pathophysiology and 
reveal the underlying risk factors of a young patient with long-term RD.

\section{Materials and methods}

Ethical statement. The present study adhered to the tenets of the Declaration of Helsinki. All clinical records and information were anonymized, and used only for research purposes. Written informed consent was provided by the guardians on behalf of the minor involved in the current study. The study procedures were approved by the Institutional Ethics Committee in Renmin Hospital of Wuhan University.

Patient information and tissue collection. A 15-year-old male patient was admitted to Renmin Hospital of Wuhan University (Wuhan, China) with left eye RD secondary to blunt trauma in May 2015. The patient had been treated for RD surgically $>10$ years earlier. However, it was unknown whether the RD occurred during the event of trauma or afterwards. The eye deteriorated to no light perception with undetectable intraocular pressure (IOP), and gradually resulted in ocular globe atrophy. By contrast, the corrected visual acuity of the patient's right eye was 20/20 and the IOP was $18 \mathrm{mmHg}$ (IOP normal range, $10-21 \mathrm{mmHg}$ ) (2). Anterior segment evaluation and B-scan ultrasonography of the eyes were performed. However, fundoscopy and optical coherence tomography examination on the left eye could not be successfully accessed due to a complicated cataract.

Since restoration of vision in the left eye was unlikely, enucleation of his left atrophic globe was performed for cosmetic purposes. Upon approval from the Ethics Committee, the surgical procedure was conducted and the ocular tissue was collected. In addition, normal ocular tissue from an age- and sex-matched donor was obtained post mortem from the eye bank of our hospital to serve as a control in the present study.

Histological techniques. The size of the eyeball, cornea and the diameter of the optic nerve of the atrophic and post-mortem eyes were measured by vernier caliper. Immunohistochemistry was then performed in the tissue samples as described in a previous study (18). Briefly, the posterior eyecups were fixed in fresh $4 \%$ paraformaldehyde dissolved in $0.1 \mathrm{M}$ PBS, $\mathrm{pH} 7.4$ at room temperature for $12 \mathrm{~h}$. The retina tissue samples were harvested with $6 \mathrm{~mm}$ trephine, followed by graded dehydration by the sequential immersion of the retinas in 10 and $20 \%$ sucrose for $2 \mathrm{~h}$ each time in $\mathrm{PBS}, \mathrm{pH} 7.4$ at $4^{\circ} \mathrm{C}$ and $30 \%$ sucrose overnight at $4^{\circ} \mathrm{C}$. The tissues were then embedded in optimal cutting temperature compound (Tissue-Tek; Sakura Finetek USA, Inc., Torrance, CA, USA), frozen in liquid nitrogen and vertically cut into $12-\mu \mathrm{m}$ sections using a Leica CM1900 cryostat (Leica Microsystems, Wetzlar, Germany). Retina sections were incubated overnight at $4^{\circ} \mathrm{C}$ in PBS containing $0.5 \%$ bovine serum albumin (BSA; Thermo Fisher Scientific, Inc., Waltham, MA, USA), $0.2 \%$ Triton X-100 plus normal donkey serum (Abcam, Cambridge, UK). The following day, this blocking serum was replaced with primary antibodies diluted in 5\% BSA (containing $0.2 \%$ Triton X-100) and further incubated overnight at $4^{\circ} \mathrm{C}$. A goat polyclonal antibody against short-wave-sensitive opsin 1 (OPN1SW; dilution, 1:200; cat. no. sc-14363; Santa Cruz Biotechnology Inc., Dallas, TX,

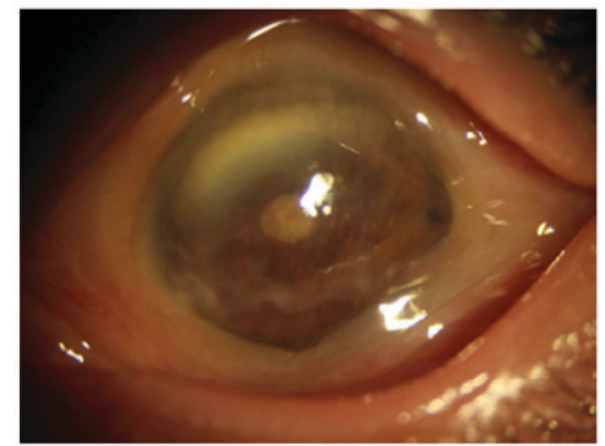

Figure 1. Anterior segment evaluation of the atrophic eye in a young patient with long-term retinal detachment demonstrated enophthalmos, leukoplakia of the cornea, pupil distortion and lens opacity.
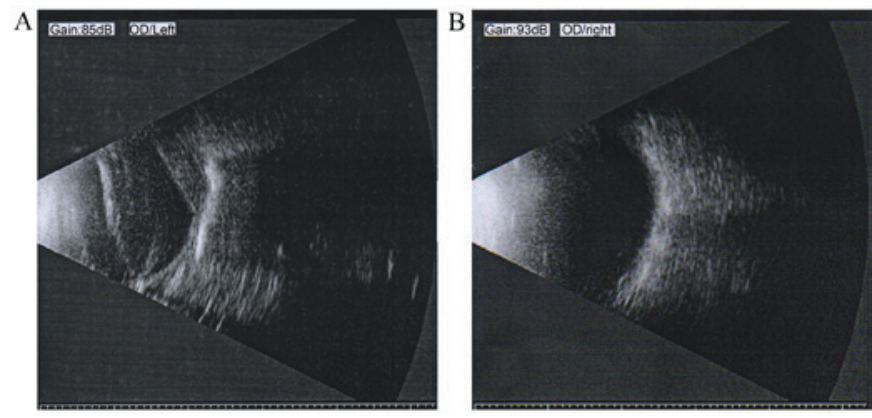

Figure 2. B-scan ultrasonography of (A) the atrophic left eye and (B) the healthy right eye of the patient. B-scan ultrasonography of the atrophic eye demonstrated long-standing retinal detachment, disorganized posterior contents and irregular posterior contour. The right eye displayed a normal appearance and clear reflection on B-scan ultrasonography.

USA) was used to label $\mathrm{S}$ cones. In addition, a mouse monoclonal antibody against glial fibrillary acidic protein (GFAP; dilution, 1:500; cat. no. G3893; Sigma-Aldrich; Merck KGaA, Darmstadt, Germany) was used to label retinal macroglia, including Müller cells and astrocytes. After three washes with PBS for $5 \mathrm{~min}$ at room temperature, the sections were incubated at $4^{\circ} \mathrm{C}$ for $2 \mathrm{~h}$ with the appropriate secondary antibodies as follows: Cy3-conjugated donkey anti-goat IgG (dilution, 1:200; cat. no. SA00009-3) and FITC-conjugated donkey anti-mouse IgG (dilution, 1:200; cat. no. SA00003-9; both ProteinTech Group, Inc., Chicago, IL, USA). Nuclear DNA was then labelled with 4',6-diamidino-2-phenylindole (DAPI). Images were captured under a fluorescence microscope (magnification, x400; BX53; Olympus Corp., Tokyo, Japan).

\section{Results}

Patient eye characteristics. The patient's left eye revealed enophthalmos, leukoplakia cornea, pupil distortion and lens opacity (Fig. 1). B-scan ultrasonography of the atrophic eye demonstrated long-term RD, disorganized posterior contents and irregular posterior contour (Fig. 2A). By contrast, B-scan ultrasonography of the unaffected right eye of the patient showed a normal appearance and clear reflection (Fig. 2B).

Eye measurements and characteristics. The size of the eyeball and cornea, and the diameter of the optic nerve of the atrophic 
Table I. Measurements of atrophic and the age- and sex-matched control eye.

\begin{tabular}{|c|c|c|c|c|c|c|}
\hline \multirow[b]{2}{*}{ Samples } & \multicolumn{3}{|c|}{ Eyeball diameter (mm) } & \multicolumn{2}{|c|}{ Cornea diameter (mm) } & \multirow{2}{*}{$\begin{array}{c}\text { Retrobulbar optic } \\
\text { nerve diameter }(\mathrm{mm})\end{array}$} \\
\hline & Vertical & Horizontal & Anterior-posterior & Vertical & Horizontal & \\
\hline Atrophic eye & 19.0 & 20.0 & 18.5 & 10.0 & 8.5 & 2.5 \\
\hline Control eye & 24.0 & 23.5 & 23.5 & 11.5 & 11.0 & 3.0 \\
\hline
\end{tabular}
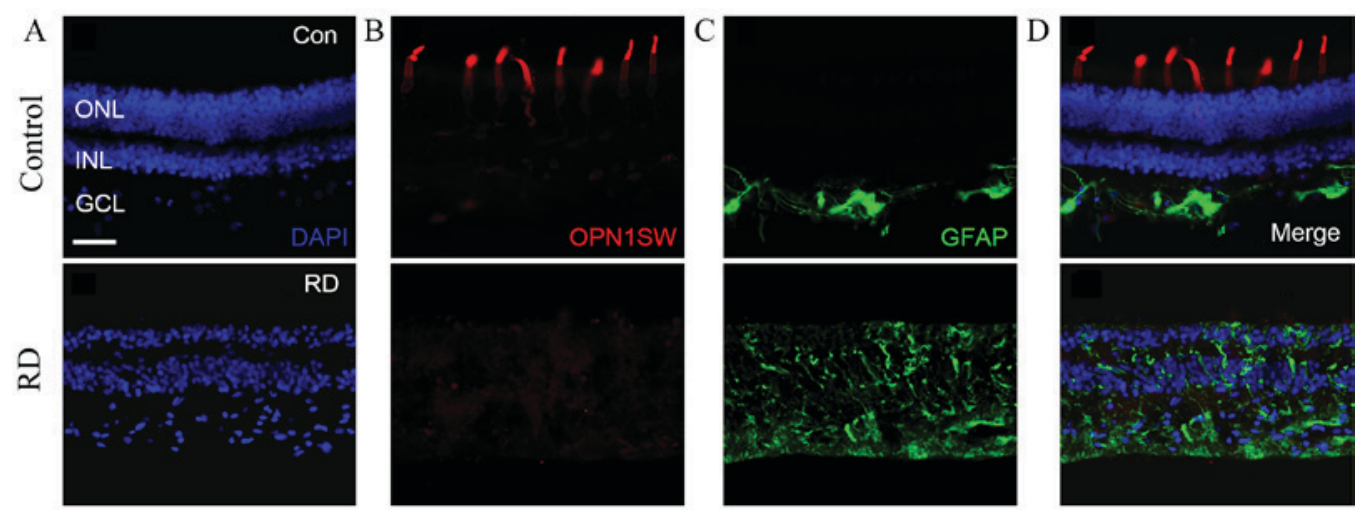

Figure 3. Expression profiles of OPN1SW and GFAP in the control and atrophic retina. (A) DAPI staining demonstrated the organized and distorted retina in the control and atrophic eyes. (B) OPN1SW staining revealed intact S cone outer segment in the control, but absent expression in the atrophic retina. (C) GFAP staining showed restricted signal of activated Müller cells in the control eye, whereas expanded signal of activated Müller cells was detected in the atrophic retina. (D) Merged images for the control and atrophic retinal tissues. Scale bar=50 $\mu \mathrm{m}$. CON, control; RD, retinal detachment; ONL, outer nuclear layer; INL, inner nuclear layer; GCL, ganglion cell layer; DAPI, 4',6-diamidino-2-phenylindole; OPN1SW, short-wave-sensitive opsin 1; GFAP, glial fibrillary acidic protein.

eye and the matched post-mortem eye were measured and compared (Table I). In the atrophic eye, the vertical, horizontal and anterior-posterior diameters of the eyeball were 19.0, 20.0 and $18.5 \mathrm{~mm}$, respectively. The horizontal and vertical corneal diameters were 10.0 and $8.5 \mathrm{~mm}$, respectively, and the diameter of the retrobulbar optic nerve was $2.5 \mathrm{~mm}$. In the healthy ocular eye, the vertical, horizontal and anterior-posterior diameters of the eyeball were $24.0,23.5$ and $23.5 \mathrm{~mm}$, respectively. The horizontal and vertical corneal diameters were 11.5 and $11.0 \mathrm{~mm}$, respectively, and the diameter of the retrobulbar optic nerve was $3.0 \mathrm{~mm}$. These results demonstrated that the ocular globe from the patient was atrophic and smaller in size compared with the size of the control eye.

When the atrophic eye was dissected vertically to obtain an eyecup, opaque vitreous with pigment deposits was observed, while the pale retina was fully detached from the posterior eyecup. Proliferation was clearly present as yellow- or white-colored linear strands in the atrophic eye. In comparison to the atrophic eye, when the matched post mortem eye was dissected, transparent gel-like vitreous humor and healthy retina was observed without any PVR signs.

Immunofluorescence analysis results. To further examine the RD-induced retina degeneration, immunofluorescence analysis was conducted in the retina tissue samples. The control retina had an intact laminar structure and organized nuclear layers as demonstrated by DAPI staining (Fig. 3A). In addition, the ratio of the outer nuclear layer (ONL) to the inner nuclear layer (INL) thickness was approximately 2:1, while the INL and ganglion cell layer (GCL) were tightly packed. Conversely, the atrophic retina displayed decreased thickness of ONL, distorted laminar structure and unorganized nuclear layers (Fig. 3A). The ONL/INL thickness ratio was approximately $1: 2$, while the INL and GCL were less tightly packed.

To identify changes of cones in the atrophic retina, outer segments of S cones were stained with OPN1SW, as reported previously (19). In the control retina, outer segment of $S$ cones were detected by dense OPN1SW staining (Fig. 3B). However, the OPN1SW signal in the atrophic retina was absent (Fig. 3B). Furthermore, GFAP staining was performed to access the gliosis of Müller cells (20) in the atrophic retina. In the control retina, sporadic GFAP staining that was likely due to astrocytes appeared primarily in the inner retina, with compressed, thin and spiky staining concentrated at the inner limiting membrane (Fig. 3C). By contrast, in the atrophic retina, evidently increased GFAP immunoactivity from Müller cell processes was detected, suggesting that increased glial cell reactivity was prominent over the entire atrophic retina (Fig. 3C). Merged images (Fig. 3D) revealed the disruption of the nuclear structure, loss of the out segment of S cone and the Müller cell gliosis in the atrophic retina.

\section{Discussion}

In the present study, a case of atrophic human retina caused by traumatic RD that occurred 10 years earlier was reported. Immunohistochemical analysis of retinal sections revealed disruption of the nuclear layer structure, loss of the $\mathrm{S}$ cone 
outer segment and hypertrophic Müller cells in the atrophic tissue. Combined with the findings of previous studies $(10,11)$, the current report provides further insight that may assist in the examination of $\mathrm{RD}$ in children in clinical practice.

Photoreceptor cell death is the ultimate cause of irreversible vision loss in RD. In previous studies, photoreceptor cell death involving Fas-receptor activation and caspase-dependent apoptosis has been widely investigated (21). Non-apoptotic types of cell death, including autophagy and necrosis, have also been reported recently $(22,23)$. A photoreceptor shift from autophagy to apoptosis is caused by photoreceptor-RPE separation in RD; however, the underling mechanisms remain unknown (24). Other areas, such as the regulatory role of mitochondria and the inflammation mechanisms during RD, also warrant further investigation (5).

In the current study, OPN1SW and GFAP staining were performed to highlight the outer segment of S cone and the gliosis in atrophic retina. Previously, the loss of S cone and the activation of glial cells have been reported in human retinal detachment $(4,7,9,24)$. The degeneration of the rod was not part of the present study, but it has previously been described in retinal detachment (9). Future work may explore the changes of the rod and other types of cones, including $\mathrm{M}$ and $\mathrm{L}$ cone, in long-term RD. In addition, other changes in RD including synaptic terminals of photoreceptors, mitochondria or various retinal neurons may be confirmed by future studies. Findings may contribute to analysis of pathological changes in long-term RD $(2,3)$. The combined analysis and targeting of photoreceptor death and Müller cell gliosis may facilitate the design of neuroprotective strategies for photoreceptors following RD.

PVR remains one of the most common causes of failed RD surgery. Müller cells respond to various pathologic stimuli in the retina, including retinitis pigmentosa, Leber congenital amaurosis, ischemia, RD, glaucoma and diabetic retinopathy (25-27). In addition, proliferating glia may serve both beneficial and harmful roles in RD (28). Detachment of the retina activates Müller cells to secrete cytokines and ATP, which do not only protect the photoreceptors, but also promote inflammation, retinal ischemia, cell proliferation and tissue remodeling $(13,29)$. Müller cell nuclei also produce intermediate filaments as a 'track' for their migration into the outer retina (12). This event, known as gliosis, perturbs the cellular layers, particularly the ONL. In an experimental RD model, the growth of Müller cells formed a fibrotic layer into the subretinal space, which completely inhibited the regeneration of the cone outer segment (30). Although Müller glial cells have the potential to proliferate and generate neurons, these responses are insufficient to repair a damaged retina (31).

The level or the 'beneficial window' of Müller cell activation may vary between pediatric and adult patients, thus requiring different management strategies. In a human study, retinas of elderly subjects demonstrated higher GFAP immunoreactivity and increased glial filaments when compared with the younger subjects (32). Furthermore, exacerbated glial response was observed in the aged mouse hippocampus following controlled cortical impact injury (33). In an experimental model of ischemia/reperfusion, retinas of old rats were more susceptible to cell death induced by secondary glial mechanisms in comparison with those of the younger rats (34). Thus, age is an important factor to be considered in the subretinal gliosis following RD.

The present study reported interesting clinical observations and immunohistochemical evaluation of RD tissue in a young male patient. The loss of the photoreceptor nucleus was observed in the atrophic eye, as well as the expansion of the INL; as a result, the ONL/INL ratio was reduced. The INL expansion can be attributed to not only the hypertrophy of Müller cell, but also the interaction between Müller cell processes and retinal neurons. As Müller cells may use intermediate filaments as a 'track' for migration, retinal neurons may possibly be stretched and migrate along the same 'track'. Further studies on the interaction between retinal neurons and Müller cells at the molecular and cellular levels may provide valuable insight into preventing photoreceptor degeneration and vision loss.

In conclusion, the present study provided an unusual opportunity to examine the characteristics of long-term traumatic $\mathrm{RD}$ in a young patient. It is suggested that greater cooperation between basic researchers and clinicians is required to match the different clinical scenarios, including age and etiology, with the biological markers of RD. Therapies to prolong photoreceptor survival may be greatly beneficial in improving the visual outcomes. Medicine treatment to prevent reactive gliosis would also be highly desirable in children with RD.

\section{Acknowledgements}

The authors would like to thank Qinqin Deng for the critical review of the manuscript.

\section{Funding}

The present study was supported by the National Natural Science Foundation of China (grant no. 81470628).

\section{Availability of data and materials}

All data generated or analyzed during this study are included in this published article.

\section{Authors' contributions}

$\mathrm{ZC}, \mathrm{YX}$ and YS conceived and designed the experiments. SL and YC performed the experiments. SL and YS analyzed the data and prepared the manuscript. All authors read and approved the final version of the manuscript.

\section{Ethics approval and consent to participate}

The study procedures were approved by the Institutional Ethics Committee in Renmin Hospital of Wuhan University. Written informed consent was provided by the guardians on behalf of the minor involved in the current study. The present study adhered to the tenets of the Declaration of Helsinki.

\section{Patient consent for publication}

Written informed consent for the publication of any associated data and accompanying images was obtained from the guardians on behalf of the minor involved in the current study. 


\section{Competing interests}

The authors declare that they have no competing interests.

\section{References}

1. Qi Y, Zhang FY, Peng GH, Zhu Y, Wan GM, Wang WZ, Ma J and Ren SJ: Characteristics and visual outcomes of patients hospitalized for ocular trauma in central China: 2006-2011. Int J Ophthalmol 8: 162-168, 2015.

2. Ghazi $\mathrm{N}$ and Green WR: Pathology and pathogenesis of retinal detachment. Eye (Lond) 16: 411-421, 2002.

3. Haimann MH, Burton TC and Brown CK: Epidemiology of retinal detachment. Arch Ophthalmol 100: 289-292, 1982.

4. Lo AC, Woo TT, Wong RL and Wong D: Apoptosis and other cell death mechanisms after retinal detachment: Implications for photoreceptor rescue. Ophthalmologica 226 (Suppl 1): S10-S17, 2011.

5. Murakami Y, Notomi S, Hisatomi T, Nakazawa T, Ishibashi T, Miller JW and Vavvas DG: Photoreceptor cell death and rescue in retinal detachment and degenerations. Prog Retin Eye Res 37: 114-140, 2013

6. Chang CJ, Lai WW, Edward DP and Tso MO: Apoptotic photoreceptor cell death after traumatic retinal detachment in humans. Arch Ophthalmol 113: 880-886, 1995.

7. Arroyo JG, Yang L, Bula D and Chen DF: Photoreceptor apoptosis in human retinal detachment. Am J Ophthalmol 139: 605-610, 2005

8. Brazitikos PD: The expanding role of primary pars plana vitrectomy in the treatment of rhegmatogenous noncomplicated retinal detachment. Semin Ophthalmol 15: 65-77, 2000

9. Nork TM, Millecchia LL, Strickland BD, Linberg JV and Chao GM: Selective loss of blue cones and rods in human retinal detachment. Arch Ophthalmol 113: 1066-1073, 1995.

10. Anderson DH, Guerin CJ, Erickson PA, Stern WH and Fisher SK: Morphological recovery in the reattached retina. Invest Ophthalmol Vis Sci 27: 168-183, 1986.

11. Rex TS, Fariss RN, Lewis GP, Linberg KA, Sokal I and Fisher SK: A survey of molecular expression by photoreceptors after experimental retinal detachment. Invest Ophthalmol Vis Sci 43: 1234-1247, 2002.

12. Lewis GP, Chapin EA, Luna G, Linberg KA and Fisher SK: The fate of Muller's glia following experimental retinal detachment Nuclear migration, cell division, and subretinal glial scar formation. Mol Vis 16: 1361-1372, 2010.

13. Garweg JG, Tappeiner C and Halberstadt M: Pathophysiology of proliferative vitreoretinopathy in retinal detachment. Surv Ophthalmol 58: 321-329, 2013.

14. Wenick AS and Barañano DE: Evaluation and management of pediatric rhegmatogenous retinal detachment. Saudi J Ophthalmol 26: 255-263, 2012.

15. Soliman MM and Macky TA: Pediatric rhegmatogenous retinal detachment. Int Ophthalmol Clin 51: 147-171, 2011.

16. Häring $\mathrm{G}$ and Wiechens B: Long-term results after scleral buckling surgery in uncomplicated juvenile retinal detachment without proliferative vitreoretinopathy. Retina 18: 501-505, 1998

17. Moisseiev J, Vidne O and Treister G: Vitrectomy and silicone oil injection in pediatric patients. Retina 18: 221-227, 1998.
18. Chen YY, Liu SL, Hu DP, Xing YQ and Shen Y: $\mathrm{N}$-methyl-N-nitrosourea-induced retinal degeneration in mice. Exp Eye Res 121: 102-113, 2014.

19. Gaillard F, Kuny S and Sauvé Y: Topographic arrangement of S-cone photoreceptors in the retina of the diurnal Nile grass rat (Arvicanthis niloticus). Invest Ophthalmol Vis Sci 50: 5426-5434, 2009.

20. Lewis GP, Erickson PA, Kaska DD and Fisher SK: An immunocytochemical comparison of Muller cells and astrocytes in the cat retina. Exp Eye Res 47: 839-853, 1988.

21. Trichonas G, Murakami Y, Thanos A, Morizane Y, Kayama M, Debouck CM, Hisatomi T, Miller JW and Vavvas DG: Receptor interacting protein kinases mediate retinal detachment-induced photoreceptor necrosis and compensate for inhibition of apoptosis. Proc Natl Acad Sci USA 107: 21695-21700, 2010.

22. Dong K, Zhu H, Song Z, Gong Y, Wang F, Wang W, Zheng Z, Yu Z, Gu Q, Xu X and Sun X: Necrostatin-1 protects photoreceptors from cell death and improves functional outcome after experimental retinal detachment. Am J Pathol 181: 1634-1641, 2012.

23. Besirli CG, Chinskey ND, Zheng QD and Zacks DN: Autophagy activation in the injured photoreceptor inhibits fas-mediated apoptosis. Invest Ophthalmol Vis Sci 52: 4193-4199, 2011.

24. Chinskey ND, Zheng QD and Zacks DN: Control of photoreceptor autophagy after retinal detachment: The switch from survival to death. Invest Ophthalmol Vis Sci 55: 688-695, 2014.

25. Bringmann A, Iandiev I, Pannicke T, Wurm A, Hollborn M, Wiedemann P, Osborne NN and Reichenbach A: Cellular signaling and factors involved in Muller cell gliosis: Neuroprotective and detrimental effects. Prog Retin Eye Res 28: 423-451, 2009.

26. Singh RK, Kolandaivelu S and Ramamurthy V: Early alteration of retinal neurons in Aipl1-/-animals. Invest Ophthalmol Vis Sci 55: 3081-3092, 2014.

27. Jacobson SG and Cideciyan AV: Treatment possibilities for retinitis pigmentosa. N Engl J Med 363: 1669-1671, 2010.

28. Bringmann A, Pannicke T, Grosche J, Francke M, Wiedemann P Skatchkov SN, Osborne NN and Reichenbach A: Müller cells in the healthy and diseased retina. Prog Retin Eye Res 25: 397-424, 2006.

29. Reichenbach A and Bringmann A: New functions of Müller cells. Glia 61: 651-678, 2013.

30. Lewis GP and Fisher SK: Müller cell outgrowth after retinal detachment: Association with cone photoreceptors. Invest Ophthalmol Vis Sci 41: 1542-1545, 2000.

31. Goldman D: Müller glial cell reprogramming and retina regeneration. Nat Rev Neurosci 15: 431-442, 2014.

32. Ramirez JM, Ramirez AI, Salazar JJ, de Hoz R and Trivino A: Changes of astrocytes in retinal ageing and age-related macular degeneration. Exp Eye Res 73: 601-615, 2001.

33. Sandhir R, Onyszchuk G and Berman NE: Exacerbated glial response in the aged mouse hippocampus following controlled cortical impact injury. Exp Neurol 213: 372-380, 2008.

34. Kim KY, Ju WK and Neufeld AH: Neuronal susceptibility to damage: Comparison of the retinas of young, old and old/caloric restricted rats before and after transient ischemia. Neurobiol Aging 25: 491-500, 2004.

This work is licensed under a Creative Commons

Attribution-NonCommercial-NoDerivatives 4.0 International (CC BY-NC-ND 4.0) License. 